\title{
Line-narrowing transient Raman technique which resolves closely spaced hydrogen-bonded aggregates
}

\author{
W. Zinth, M. C. Nuss, and W. Kaiser \\ Physik Department der Technischen Universität München, \\ D-8000 München, Germany \\ (Received 11 May 1984)
}

\begin{abstract}
An ultrafast coherent Raman spectroscopy with tunable excitation is presented. It is applied to the analysis of vibrational spectra of hydrogen-bonded aggregates of pyridine and methanol. Whereas the spontaneous Raman spectrum exhibits a broad and featureless band, the line-narrowed spectra reveal three distinct lines. The concentration dependence of these lines suggests an assignment to well-defined aggregates of different sizes.
\end{abstract}

In congested spectral regions homogeneously broadened lines frequently overlap to such an extent that the different transition frequencies and amplitudes may not be resolved in the spontaneous spectrum. We present here a promising coherent spectroscopic method, which reduces the homogeneous width of the transition and reveals the inhomogeneous frequency distribution.

Several previous publications have shown that spectral resolution may be improved in transient experiments, where resolution is not limited by the lifetime of the observed levels but by the specific experimental conditions. ${ }^{1-4}$

In this Rapid Communication we study Raman active molecular vibrations in liquids by means of short excitation and prolonged interrogation (SEPI). A short and transient excitation process generates a coherent vibrational amplitude. Subsequently, a delayed and suitably (e.g., Gaussian) shaped probing pulse interrogates the coherently vibrating molecules. For sufficiently long interrogation times the spectral resolution is improved. In previous experiments only a small number of discrete excitation frequencies was available limiting the applicability of the line-narrowing technique. $^{3}$ Here, we introduce an experimental system with smooth tunability of the excitation enabling us to determine frequency positions and amplitudes of individual transitions in highly congested spectral regions.

First we present the theoretical description of the linenarrowing technique and derive expressions essential for the determination of transient amplitudes. We treat a system of closely spaced vibrational transitions at frequencies $\omega_{j}$ with Raman cross sections $\sigma_{j}$ and with dephasing times $T_{2 j}$. The excitation force $\tilde{A}(t)=A(t) \cos \left(\omega_{0} t\right)$ is proportional to the product of two incident light fields, $E_{L} E_{S}^{*}$, with $\omega_{0}=\omega_{L}-\omega_{S} ; \tilde{A}(t)$ is applied for the short time $t_{p E}$. The excitation of the coherent amplitude $q_{j}=\frac{1}{2}\left[Q_{j}\right.$ $\times \exp \left(-i \omega_{j} t\right)+$ c.c.] follows the equation of motion: ${ }^{5,6}$

$$
\frac{\partial^{2} q_{j}}{\partial t^{2}}+\frac{2}{T_{2 j}} \frac{\partial q_{j}}{\partial t}+\omega_{j}^{2} q_{j}=\tilde{A}(t)
$$

with the solution

$$
\begin{array}{rl}
Q_{j}(t)=\frac{i}{2 \omega_{j}} \exp \frac{-t}{T_{2 j}} \int_{-\infty}^{t} & d t^{\prime} A\left(t^{\prime}\right) \\
& \times \exp \left[\left(\frac{1}{T_{2 j}}+i \Delta \omega_{j}\right) t^{\prime}\right] .
\end{array}
$$

$\Delta \omega_{j}=\omega_{j}-\omega_{0}$ represents the frequency difference between the individual transition $j$ and the driving force. At late times $t>t_{p E}$ the coherent amplitude $q_{j}$ oscillates at its resonance frequency $\omega_{j}$ and decays exponentially with the dephasing time $T_{2 j}$. With increasing off-resonance $\left|\Delta \omega_{j}\right|$ the amplitude $\left|Q_{j}\right|$ becomes smaller.

The salient feature of the line-narrowing technique is the prolonged interrogation. The coherently oscillating molecules are probed by a second laser field

$$
\tilde{E}_{L 2}\left(t-t_{D}\right)=\frac{1}{2}\left[E_{L 2}\left(t-t_{D}\right) \exp \left(-i \omega_{L 2} t\right)+\text { c.c. }\right]
$$

which is delayed by $t_{D}$. The propagation directions of the exciting and probing beams in the sample are adjusted in order to obtain phase matching for coherent anti-Stokes scattering. ${ }^{6}$ The anti-Stokes field $E_{A}$ is produced according to Eq. (3):

$$
\frac{\partial E_{A}}{\partial x} \propto \sum_{j} N_{j} \sigma_{j} E_{L 2}\left(t-t_{D}\right) Q_{j}(t) \exp \left[-i\left(\omega_{L 2}+\omega_{j}\right) t\right] \text {. }
$$

$N_{j}$ is the particle density of the component $j$. We use a Gaussian-shaped probing pulse with

$$
E_{L 2}(t)=E_{L 2 O} \exp \left[-\left(t / t_{p}\right)^{2} 2 \ln 2\right] .
$$

When a single vibrational component $j$ is excited by a resonant Gaussian force one calculates an anti-Stokes spectrum $I_{A}(\Omega)$ at late delay times $t_{D}>t_{p}$.

$$
\begin{aligned}
I_{A}(\Omega) \propto & N_{j}^{2} \sigma_{j}^{2} \exp \left[\left(\frac{t_{p E}^{2}}{2}+t_{p}^{2}\right) /\left(4 \ln 2 T_{2 j}^{2}\right)-\frac{2 t_{D}}{T_{2 j}}\right] \\
& \times \exp \left[-\left(\frac{\left(\Omega-\Omega_{A}\right)^{2}}{4 \ln 2 / t_{p}^{2}}\right)\right] .
\end{aligned}
$$

According to Eq. (4) the Raman line at the anti-Stokes frequency $\Omega_{A}=\omega_{L 2}+\omega_{j}$ has a Gaussian shape and a width $\Delta \nu_{\mathrm{SEPI}}=2 \ln 2 / \pi t_{p}=0.44 / t_{p}$ which is determined by the duration of the probing pulse and not by the molecular dephasing time. When the probing pulse is sufficiently long, i.e., for $t_{p} \geq 1.4 T_{2}$, the observed SEPI line is narrower than the spontaneous Raman spectrum with $\Delta \nu_{\text {spont }}=1 / \pi T_{2}$. The amplitude of the SEPI spectrum depends on experimental parameters (durations of excitation $t_{p E}$ and of probing pulses $t_{p}$ ) and on molecular quantities (dephasing time $T_{2 j}$ and Raman cross sections $\sigma_{j}$ ). $T_{2 j}$ can be determined by measuring the SEPI spectra at different delay times, while $\sigma_{j}$ can be deduced from the peak height of the spectra.

For strongly overlapping transitions the coherent spec- 
trum has to be calculated from Eq. (3). Inspection of Eq. (3) shows that the resolution of the components may be optimized by proper choice of the delay time $t_{D}$. In the present experiment $t_{D}$ was chosen to be $15 \mathrm{ps}$. A comment should be made to the shape of the probing pulses. In our calculation we used a Gaussian-shaped pulse providing a substantial reduction of the spectral width. For other shapes of the probing pulse the line narrowing must be determined by solving Eq. (3).

Vibrational modes in liquids at room temperature have phase relaxation times $T_{2}$ of the order of $10^{-12} \mathrm{~s}$. Consequently, one needs picosecond pulses for transient experiments. In the SEPI technique we use a pair of picosecond pulses with tunable frequency difference for the excitation and a third pulse (which is a part of one exciting pulse) for the prolonged probing. A schematic of the experimental system is depicted in Fig. 1. Pulses from a mode-locked argon-ion laser synchronously pump two dye lasers. The first dye laser SML I (dye rhodamine 6G, three-plate birefringent filter, ethalon) emits nearly bandwidth limited pulses of $12 \mathrm{ps}$ duration at $\tilde{\nu}_{L}=16550 \mathrm{~cm}^{-1}$. The second dye laser SML II (dye DCM, three-plate birefringent filter) generates pulses of 6 ps duration tuned between 15500 and $15600 \mathrm{~cm}^{-1}$ in our investigations. The dye laser pulses are synchronized in time with a jitter of less than 5 ps. The two laser pulses simultaneously traverse the sample generating a transient coherent molecular vibration. The optical delay line (OD1) is used to optimize the temporal overlap of the exciting pulses. Part of the beam from SML I is used for the probing process $\left(\tilde{\nu}_{L 2}=\tilde{\nu}_{L}\right)$ with a delay time of $t_{D}=15$ ps. The different beams cross in the sample in a geometry appropriate for coherent anti-Stokes scattering. The anti-

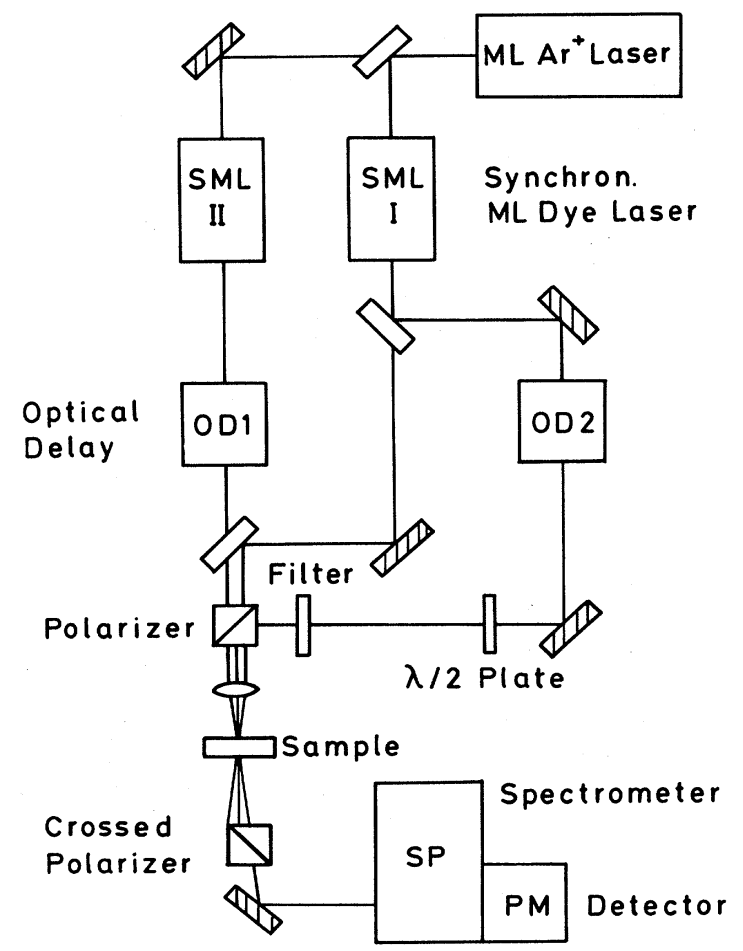

FIG. 1. Schematic of the "spectrometer" used for line-narrowing transient Raman spectroscopy. A cw mode-locked argon-ion laser pumps two synchronously mode-locked dye lasers which serve as excitation and probing light sources.
Stokes radiation of the probing process passes the second polarizer and is detected with the help of the spectrometer (resolution $0.5 \mathrm{~cm}^{-1}$ ). In the present experiments the Stokes frequency $\tilde{\nu}_{S}$ is varied in steps of $1 \mathrm{~cm}^{-1}$. For each value of $\tilde{\nu}_{S}$ the coherent anti-Stokes spectrum was measured. In this way we take advantage of the line narrowing due to prolonged interrogation and of the suppression of lines by off resonance excitation.

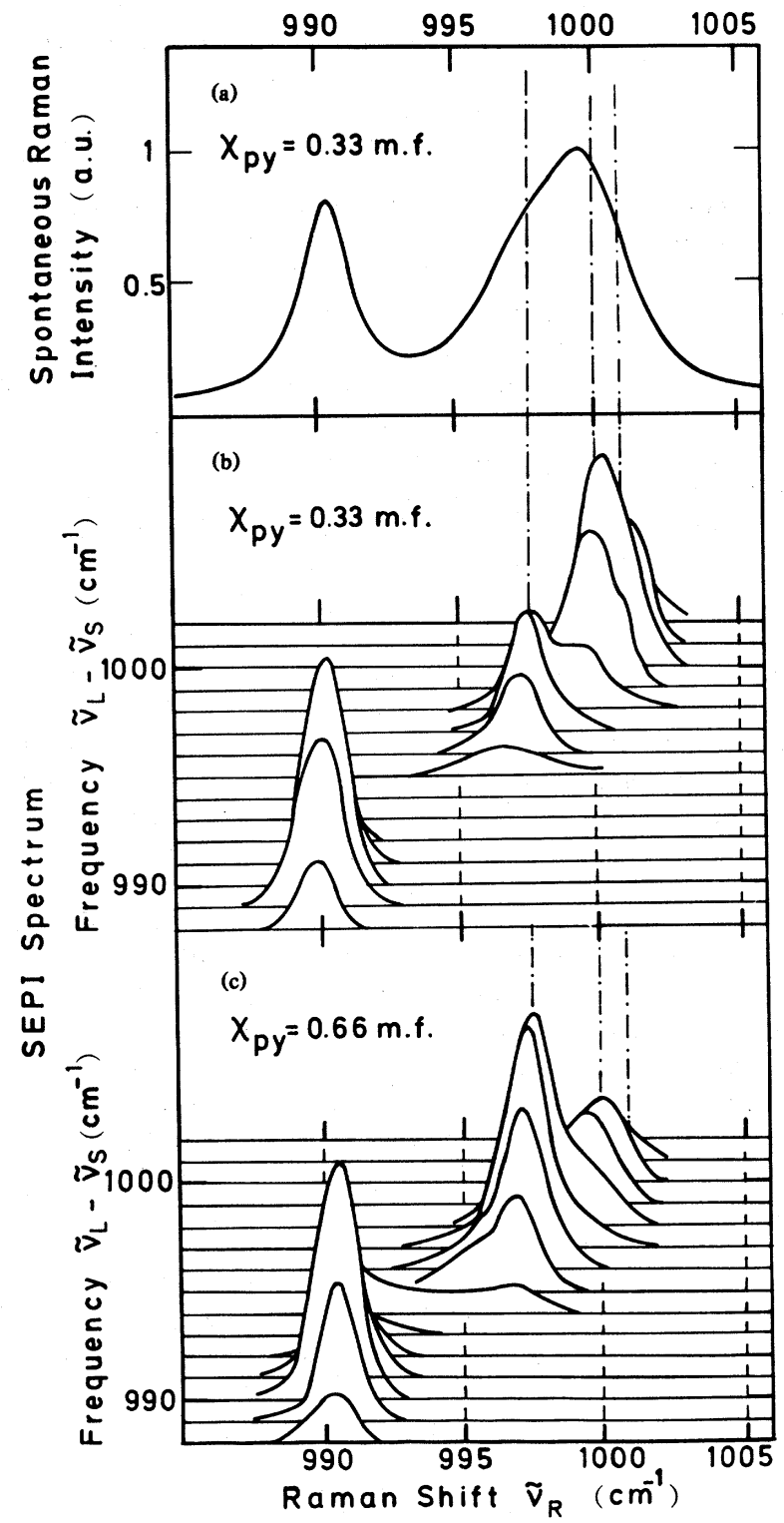

FIG. 2. Raman spectra of pyridine-methanol mixtures. (a) Polarized spontaneous Raman spectrum measured at a pyridine concentration of 0.33 molar fraction taken with a standard Raman spectrometer of spectral resolution of $0.5 \mathrm{~cm}^{-1}$. (b) and (c) Short excitation and prolonged interrogation (SEPI) spectra obtained for a set of excitation frequencies $\tilde{\nu}_{L}-\tilde{\nu}_{S}$ at two pyridine concentrations, $\chi_{p y}=0.33$ molar fraction (b) and $\chi_{p y}=0.66$ molar fraction (c). The spectra, taken at excitation frequencies $\tilde{\nu}_{L}-\tilde{\nu}_{S}<994 \mathrm{~cm}^{-1}$, are drawn with reduced amplitudes $[\times 0.25$ in (b) and $\times 0.02$ in (c)]. The SEPI spectra resolve three Raman lines of different hydrogenbonded aggregates hidden under the broad band of the spontaneous Raman spectrum (see dash-dotted lines) 
In Fig. 2 we present experimental data. A spontaneous Raman spectrum is compared with two SEPI spectra. The spontaneous spectrum of Fig. 2(a), taken of a pyridinemethanol mixture with $\chi_{p y}=0.33$ molar fraction, shows two bands. The smaller band at $990 \mathrm{~cm}^{-1}$ corresponds to the ring mode $\nu_{1}$ of pyridine. ${ }^{7}$ The broad $\left(\sim 6 \mathrm{~cm}^{-1}\right)$ and asymmetric band at $1000 \mathrm{~cm}^{-1}$ is assigned to hydrogenbonded complexes. ${ }^{8}$

Completely different are the spectra obtained with our coherent SEPI technique. In Figs. 2(b) and (c) a series of spectra is presented which were taken at different excitation frequencies $\nu_{L}-\nu_{S}$. The situation is straightforward for the strong band at $990.2 \mathrm{~cm}^{-1}$, which has a peak position nearly independent of the excitation frequency. This line corresponds to the pyridine molecules not involved in hydrogen bonding with methanol.

Of special interest here are the narrow SEPI spectra for a Raman shift of approximately $1000 \mathrm{~cm}^{-1}$. We find two distinct bands at $997.3 \pm 0.5 \mathrm{~cm}^{-1}$ and at $1000.0 \pm 0.5 \mathrm{~cm}^{-1}$ and a weaker component at $1001.0 \pm 0.5 \mathrm{~cm}^{-1}$. The three frequency positions are marked in Fig. 2 by vertical dashdotted lines. The relative amplitudes of the two major components change considerably with concentration. Whereas the component at $1000 \mathrm{~cm}^{-1}$ is the strongest at $\chi_{p y}=0.33$ molar fraction [see Fig. 2(b)], one finds the component at
$997.3 \mathrm{~cm}^{-1}$ to dominate at $\chi_{p y}=0.66$ molar fraction [see Fig. 2(c)]. A large number of SEPI spectra were taken for pyridine concentrations between $\chi_{p y}=0.1$ and 1.0 molar fraction. The band at $997.3 \mathrm{~cm}^{-1}$ was found to rise to a maximum value at $\chi_{p y}=0.5$ molar fraction, while the band at $1000 \mathrm{~cm}^{-1}$ showed a maximum at higher methanol concentrations with $\chi_{p y}=0.25$ molar fraction.

Our data suggest the following picture for the hydrogenbonded complexes: (i) There exist three distinct complexes of pyridine and methanol. (ii) The species at $997.3 \mathrm{~cm}^{-1}$ seems to consist of one pyridine and one methanol molecule, PM. These complexes have their highest concentration when pyridine and methanol are in equal numbers in the solution. (iii) The component at $1000 \mathrm{~cm}^{-1}$ appears to be made up of one pyridine and two or-more probablyfour methanol molecules, $\mathrm{PM}_{2}$ or $\mathrm{PM}_{4}$. We note that selfassociation of methanol occurs at high methanol concentrations with a predominance of methanol tetramers. ${ }^{9}$ (iv) The component at $1001 \mathrm{~cm}^{-1}$ is tentatively assigned to complexes $\mathrm{PM}_{n}$ with $n>4$.

In summary, we can say that the presented coherent Raman technique allows to resolve Raman lines where the spontaneous Raman spectrum exhibits a broad and featureless band. The frequency positions and the relative amplitudes of the individual transitions are readily determined.
${ }^{1}$ F. J. Lynch, R. E. Holland, and M. Hamermesh, Phys. Rev. 120, 513 (1960); C. S. Wu, Y. K. Lee, N. Benczer-Koller, and P. Simms, Phys. Rev. Lett. 5, 432 (1960).

2I.-J. Ma, J. Mertens, G. zu Putlitz, and G. Schütte, Z. Phys. 208, 352 (1968); G. Copley, B. P. Kibble, and G. W. Series, J. Phys. B 1, 724 (1968); H. Figger and H. Walther, Z. Phys. 267, 1 (1974).

${ }^{3}$ W. Zinth, Opt. Commun. 34, 479 (1980); W. Zinth, M. C. Nuss, and W. Kaiser, Chem. Phys. Lett. 88, 257 (1982).

${ }^{4}$ W. Zinth, M. C. Nuss, and W. Kaiser, Opt. Commun. 44, 262 (1983).
${ }^{5}$ R. L. Carman, F. Shimizu, C. S. Wang, and N. Bloembergen, Phys. Rev. A 2, 60 (1970).

6J. A. Giordmaine and W. Kaiser, Phys. Rev. 144, 676 (1966).

${ }^{7}$ D. A. Long, F. S. Murfin, and E. L. Thomas, Trans. Faraday Soc. 59, 12 (1963).

${ }^{8}$ H. Takahashi, K. Mamola, and E. K. Plyler, J. Mol. Spectrosc. 21, 217 (1966); B. P. Asthana, H. Takahashi, and W. Kiefer, Chem. Phys. Lett. 94, 41 (1983).

${ }^{9}$ W. B. Dixon, J. Phys. Chem. 74, 1396 (1970); A. N. Fletcher, ibid. 75, 1808 (1971). 